\title{
Guidance of Visual Search by Preattentive Information
}

\author{
Jeremy M. Wolfe
}

\begin{abstract}
When searching for one object among distracting objects, observers make use of a limited set of stimulus attributes to guide the deployment of their attention. Thus, if asked to search for ripe strawberries, an observer will use color information to guide covert attention as well as overt movements of eye and hand. While color is an unambiguous source of such guidance, there is no universally agreed list of all such sources. This chapter reviews the methods for determining if a property can guide the deployment of attention. Candidate sources of guidance are listed found in a table.
\end{abstract}

\section{INTRODUCTION}

Many aspects of visual processing operate in parallel. The retina, to offer an obvious example, handles input from the entire visual field at one time. Early vision continues this parallel processing. Much farther along in the stream of processing, recognizing an object must involve comparing the visible object with many stored representations in parallel. It is implausible to suppose that the visible object could be matched to all of the possible stored representations in series. However, between the early reception of visual input and the later recognition of visible objects, there is a chokepoint (perhaps more than one). We cannot recognize all of the objects in the visual field at once. At any moment in time, a limited subset of the initial parallel processing of the visual input, perhaps a single object, must be selected for delivery to parallel object recognition processes. That act of selection is not random. Information that is made available by initial parallel processing can be used to control selection.
Thus, if we are looking for our red car in the parking lot, we select red cars to examine, not blue cars.

If we accept this set of propositions, several terms can be defined. Visual attention can be said to be guided by features (e.g., "red") of the visual stimulus. Those features can be said to be preattentive in the sense that the visual information about the feature must have become available before the act of selection to guide that selection. It is useful to reserve the term preattentive features for specific values (e.g., "red") on a preattentive dimension (in this case, "color"). Two types of guidance can be distinguished. If an item differs from its neighbors in a preattentive dimension, that difference, itself, can attract attention in a "bottom-up," stimulus-driven manner. If the observer is told to look for targets having a specific preattentive feature, attention can be guided to items having that feature in a "top-down" manner. Only a limited amount of visual information is available preattentively to guide selective attention. This article briefly reviews the nature of that preattentive guidance.

\section{LOOKING FOR PREATTENTIVE FEATURES}

Preattentive features can be identified by several experimental tests. No one test is perfectly definitive. Ideally, a series of converging operations support the designation of a feature, though, in practice, quite a few features and dimensions are on the list on the basis of rather few data. Much evidence comes from visual search tasks where an observer looks for a target item among a number of distracting items. Typically, reaction time (RT) and / or accuracy are measured as a function of the set size (the number of items). If the target is defined by a unique preattentive feature, then the 


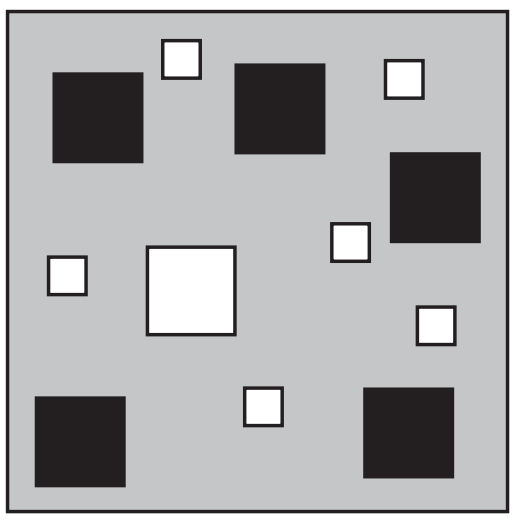

FIGURE 17.1 Find the big white target.

slope of these functions should be near zero. That is, attention should be guided to the target item with minimal interference from distracting items. Thus, search such as red among green, vertical among horizontal, moving among stationary, and big among small will typically produce $\mathrm{RT} \times$ set size slopes near zero, indicating that color, orientation, motion, and size are dimensions that are available to guide attention (Treisman, 1988; Treisman and Gelade, 1980).

RT $x$ set size slopes near zero are not definitive marks of a basic feature because other search tasks can produce such slopes. For example, a search for a conjunction of two features can produce highly efficient slopes if each of the two features is sufficiently salient. In Fig. 17.1, the big white target is found efficiently, not because big whiteness is a feature but because the attention is guided to white and to big and the intersection of those two sources of guidance is a good marker for the likely location of big white items (Wolfe, 1994; Wolfe et al., 1989).

What other clues to preattentive status are available if slopes alone are inadequate? As Treisman first noted, search asymmetries can be useful (Treisman and Gormican, 1988; Treisman and Souther, 1985). The presence of a feature guides attention better than its absence. For example, it is easier to find a moving stimulus among stationary than a stationary item among moving. Of course, motion is a fairly obvious feature. Search asymmetry comes into its own in ambiguous cases. For example, over a range of experiments, search for the opaque item is more efficient than search for the transparent. This suggests that opacity may be a feature and that, for purposes of preattentive guidance, transparency may merely be the absence of that feature.

In some circumstances, search asymmetries may not be evidence for the presence of a guiding feature. Suppose that a search for an item A among B or B

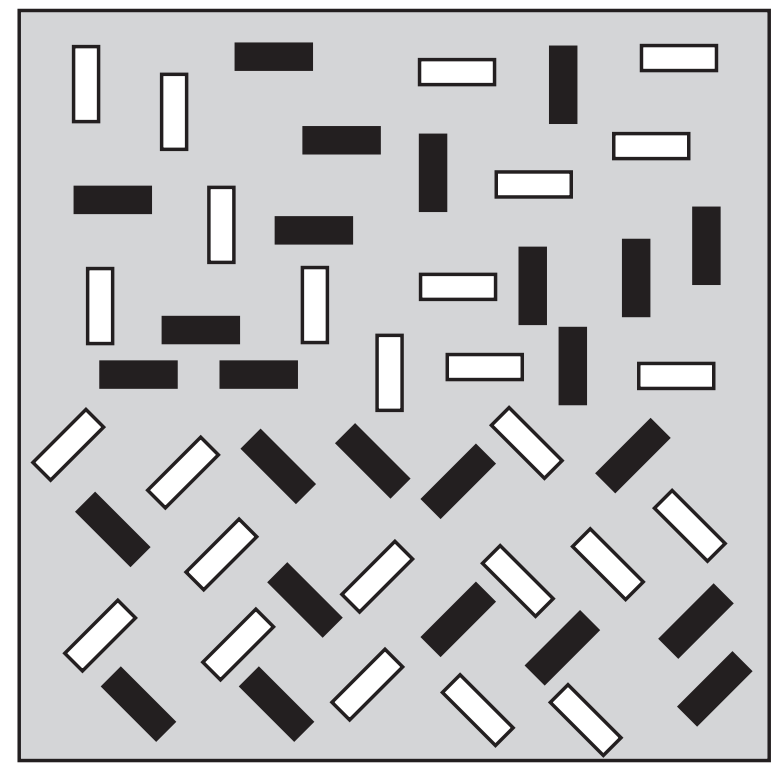

FIGURE 17.2 Where are the texture boundaries?

among $\mathrm{A}$ is unguided. Items are sampled randomly from the display. If it takes longer to reject $\mathrm{B}^{\prime} \mathrm{s}$ than it does to reject $A^{\prime}$ s, then search for $A$ among $B$ will be slower than search for B among A. For example, it can be harder to search for a "smile" curve among frowns when the critical curved line is embedded in a schematic face (Suzuki and Cavanagh, 1995). This slowing of search does not have to do with the absence of a feature but with the particularities of processing facelike stimuli.

Texture segmentation is the other most useful converging operation. The difference between texture segmentation and visual search is illustrated in Fig. 17.2. It is very clear that there is a boundary, defined by a change in orientation, dividing the upper and lower parts of the figure. It is much less obvious that there is a boundary, defined by a dramatic change in conjunctive properties, between the left and right halves of the figure. A search for white vertical or black horizontal among black vertical and white horizontal would produce efficient conjunction search (similar to Fig. 17.1), but texture boundaries defined by these conjunctions are not salient (Wolfe, 1992).

\section{HOW GUIDANCE WORKS}

Although there is little doubt that a property like orientation can be said to be preattentive, this does not mean that any detectable difference in a preattentive dimension can be used to guide attention. The guiding signals are coarse. In orientation, for example, a 
vertical line will be found very efficiently among horizontal lines but not among lines tilted $5^{\circ}$ off vertical, even though that $5^{\circ}$ difference is well above detection threshold (Foster and Ward, 1991). Guidance is a form of a signal detection problem. The target feature provides a signal and other aspects of the display generate noise (as does intrinsic noise in the nervous system) (Verghese, 2001). A feature can guide attention if it is available preattentively and if it generates a signal of adequate size. This leads to a certain ambiguity. For example, search for opaque surfaces among transparent generates RT $x$ set size functions that are on the order of $8 \mathrm{~ms} /$ item for target-present trials. This is much more efficient than the 20- to 30-ms slopes characterizing inefficient, unguided search, but $8 \mathrm{~ms}$ is not zero. The suggestion is that this stimulus property generates a relatively weak preattentive signal. Weak guidance might make search more efficient but not reduce RT x set size slopes to zero. Despite efforts to enshrine a magic value such as $10 \mathrm{~ms} /$ item, there is no sharp cutoff criterion for slope magnitude that allows us to declare in an unambiguous manner that a guiding feature is or is not present. The set of all search tasks produces a continuum of slopes, not a neat dichotomy between the efficiently guided (or "parallel") and the inefficiently guided or unguided ("serial") (Wolfe, 1998). Faced with weak evidence for a feature, the best remedies are to seek converging evidence (see above) or to design better stimuli.

Because the features that guide attention are properties that we can see and describe, it is tempting to think that these features behave in the same way when used by preattentive guidance as they do when they generate conscious, attended perception. This does not appear to be the case. In addition to being quite coarse, preattentive dimensions may be divided up differently from their perceptual counterparts. For example, orientation guidance may be "categorical. " Preattentive orientation processing seems to discriminate between "steep," "shallow," "left," and "right," rather than the more strictly geometrical angular differences that are important in making attended discriminations (Wolfe et al., 1992).

In keeping with the view of guidance as a form of signal detection, increasing the "noise" reduces the efficiency of search in the same manner as decreasing the signal. Thus, increasing the variability of the distracter items decreases the efficiency of search. For example, $0^{\circ}$ vertical among $20^{\circ}$ distracters is more efficient than $0^{\circ}$ among $20^{\circ}$ and $40^{\circ}$, even though the $40^{\circ}$ distracters are more distinct from the target. This example makes another point. There is nothing "noisy" about $20^{\circ} 40^{\circ}$ items in isolation. It is the local differences between items that seem to be the source
TABLE 17.1 Probable, Possible, and Unlikely Sources of Preattentive Guidance ${ }^{\mathrm{a}}$

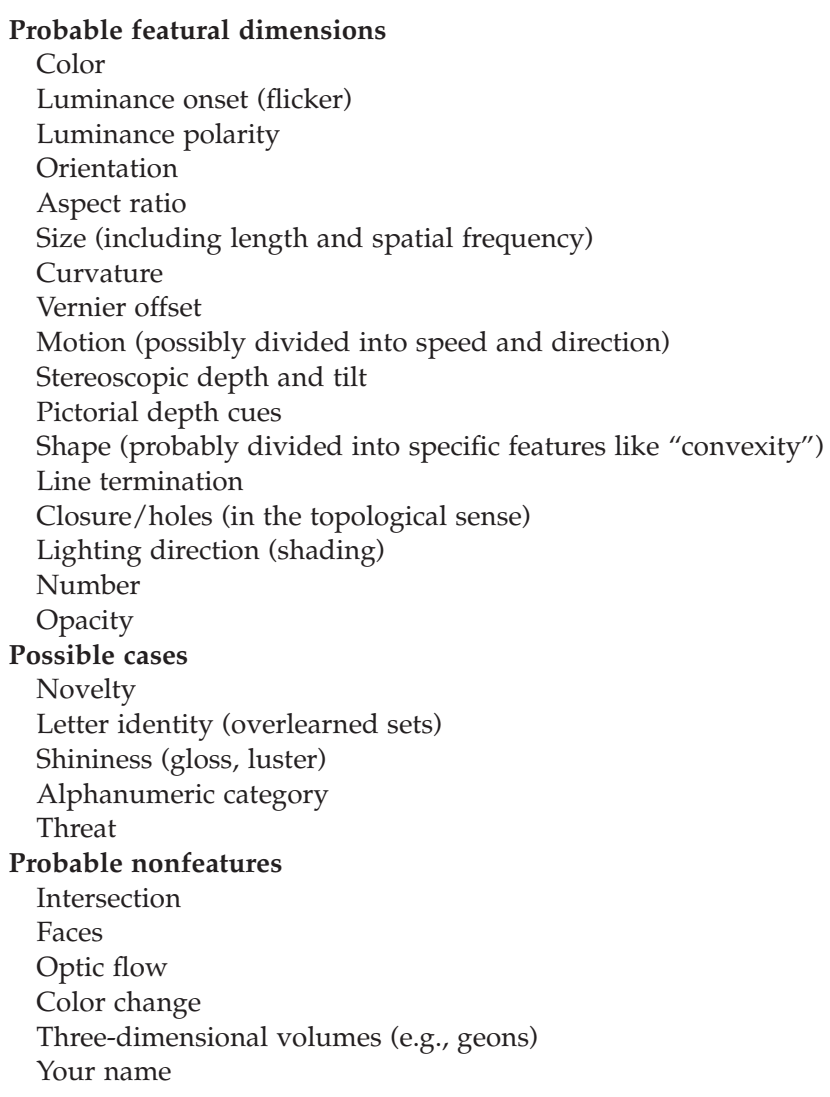

${ }^{\text {a }}$ Details and references available from the author.

of noise. Similarly, the "signal" is not the vertical item, in this example, but the local difference between the vertical item and surrounding items. Local differences are greater when items are closer together; as a consequence, the "signal" can increase with set size when the density of items increases with set size. In turn, this can produce negative RT $x$ set size slopes (Bravo and Nakayama, 1992).

\section{WHAT ARE THE PREATTENTIVE FEATURES?}

Of course, after the forgoing discussion, the obvious question is "What aspects of the visual stimulus provide preattentive guidance?" This short chapter does not provide the space for a full discussion with all of the several hundred relevant references. Table 17.1 lists "likely," "possible," and "unlikely" sources of guidance.

Many of the items on this list have been the subject of very limited amounts of study, sometimes only a 
single published paper. Consequently, it would not be a surprise if the list changed. While the details may change, the larger principle seems likely to endure. The front end of the visual system can process vast amounts of information in parallel. Later processes, like those of object recognition, can match visual input to a vast number of stored object representations, in parallel. Because it is not possible to match all of the input to all of the possible objects at the same time, selection mechanisms, guided by a set of preattentive features, govern the transfer of a limited amount of information from one massively parallel stage to the next.

\section{References}

Bravo, M., and Nakayama, K. (1992). The role of attention in different visual search tasks. Percept. Psychophys. 51, 465-472.

Foster, D. H., and Ward, P. A. (1991). Horizontal-vertical filters in early vision predict anomalous line-orientation identification frequencies. Proc. R. Soci. London Ser. B 243, 83-86.

Suzuki, S., and Cavanagh, P. (1995). Facial organization blocks access to low-level features: an object inferiority effect. J. Exp. Psychol. Hum. Percept. Perform. 21, 901-913.
Treisman, A. (1988). Features and objects: the 14th Bartlett memorial lecture. Q. J. Exp. Psychol. 40A, 201-237.

Treisman, A., and Gelade, G. (1980). A feature-integration theory of attention. Cogn. Psychol. 12, 97-136.

Treisman, A., and Gormican, S. (1988). Feature analysis in early vision: evidence from search asymmetries. Psych. Rev. 95, 15-48.

Treisman, A., and Souther, J. (1985). Search asymmetry: a diagnostic for preattentive processing of seperable features. J. Exp. Psychol. Gen. 114, 285-310.

Verghese, P. (2001). Visual search and attention: a signal detection approach. Neuron 31, 523-535.

Wolfe, J. M. (1992). "Effortless" texture segmentation and "parallel" visual search are not the same thing. Vision Res. 32, 757-763.

Wolfe, J. M. (1994). Guided Search 2.0: a revised model of visual search. Psychon. Bull. Rev. 1, 202-238.

Wolfe, J. M. (1998). What do 1,000,000 trials tell us about visual search? Psychol. Sci. 9, 33-39.

Wolfe, J. M., Cave, K. R., and Franzel, S. L. (1989). Guided search: an alternative to the feature integration model for visual search. J. Exp. Psychol. Hum. Percept. Perform. 15, 419-433.

Wolfe, J. M., Friedman-Hill, S. R., Stewart, M. I., and O'Connell, K. M. (1992). The role of categorization in visual search for orientation. J. Exp. Psychol. Hum. Percept. Perform. 18, 34-49. 